$17^{\text {th }}$ International Congress of Metrology, 06004 (2015)

DOI: $10.1051 /$ metrology / 201506004

(C) Owned by the authors, published by EDP Sciences, 2015

\title{
Gestion de la métrologie des températures dans un laboratoire de PMA (Procréation Médicalement Assistée)
}

\author{
V.LEMARQUIS, L.SERTHELON \\ LBM Billiem, France
}

\begin{abstract}
To organize the metrological follow-up of the temperatures in a laboratory of MAP (MEDICALLY ASSISTED PROCREATION) is bound (connected) to the specific nature of the realized examinations and a number of peculiarities are to be taken into account. This metrological follow-up is organized in 3 key stages.

The laboratory identifies its critical equipments, having a significant incidence on the accuracy and the reliability of the results (profits), the employees within the framework of the supervision of the conditions of realization of the examinations of MAP (MEDICALLY ASSISTED PROCREATION)

The laboratory defines the corresponding specified metrological requirements (EMT) according to the requirements of the testing method and the recommendations of the learned societies.

The laboratory puts in Implementation the metrological follow-up of the various measuring instruments and the moderate greatnesses on a daily basis.

Conclusion: importance of the management of the metrology of the temperatures in a LBM of MAP (MEDICALLY

ASSISTED PROCREATION)
\end{abstract}

Keywords: Critical equipment; connecting; metrological politics, MAP (MEDICALLY ASSISTED PROCREATION),

\section{Introduction}

L'évolution de la réglementation impose aux laboratoires de biologie médicale une accréditation complète de leurs activités d'ici fin 2020 selon la norme EN ISO 15189 [2] ; Les facteurs clés du succès de cette étape d'accréditation passent par une maîtrise des méthodes d'analyse, la mise en place d'une gestion des équipements d'essais et de mesure, et laisse une place importante à la métrologie ;

L'assistance médicale à la procréation (AMP ) s'entend des pratiques cliniques et biologiques permettant l'insémination artificielle et la conception in vitro, le transfert d'embryons ainsi que toute technique d'effet équivalent permettant la procréation en dehors du processus naturel. [6]

Le laboratoire d'AMP présente deux activités :

-une activité de spermiologie : une activité de spermiologie diagnostique avec la réalisation de spermogramme et spermocytogramme, une activité de spermiologie thérapeutique avec la réalisation d'insémination intra utérine, de fécondation in vitro, d'ICSI .

-une activité de FIV (fécondation in vitro) méthode qui permet de réaliser la fécondation à l'extérieur du corps humain en réunissant en laboratoire les ovocytes de la femme et les spermatozoides de l'homme; l'activité de
FIV se déroule en différentes phases au niveau du laboratoire:

Le transport des ovocytes du bloc au laboratoire après ponction folliculaire,

La fécondation,

Le développement des embryons,

La cryoconservation des embryons.

\section{Définition des besoins}

La métrologie mise en œuvre est définie en fonction des besoins du laboratoire, déterminés à l'aide d'une analyse de risque ou des données provenant des vérifications de méthode sur site [3] ; la politique du laboratoire est de raccorder métrologiquement que les équipements et grandeurs du laboratoire qui ont une influence sur le résultat ; le laboratoire identifie donc parmi l'ensemble de son matériel ses équipements et grandeurs critiques qui ont une influence significative sur la qualité, la fiabilité et l'exactitude du résultat final. Ceci concerne aussi bien des équipements, que d'autres types de mesure comme les conditions environnementales, ou le préanalytique. 
Figure 1 Les Equipements critiques en SPERMIOLOGIE pour la grandeur température

\begin{tabular}{|l|l|}
\hline Phase opérationnelle & Equipements critiques \\
\hline Phase préanalytique & \\
\hline Phase de liquéfaction & $\begin{array}{l}\text { Etuve }+ \text { sondes } \\
\text { indépendantes }\end{array}$ \\
\hline Phase analytique des & $\begin{array}{l}\text { Réfrigérateur }+ \text { sondes } \\
\text { indépendantes }\end{array}$ \\
\hline $\begin{array}{l}\text { Conservation } \\
\text { réactifs }\end{array}$ & Cuve d'azote \\
\hline $\begin{array}{l}\text { Phase post analytique } \\
\text { Conservation }\end{array}$ & \\
\hline \multicolumn{2}{|c|}{ postytique }
\end{tabular}

Figure 2: Les Equipements critiques en EMBRYOLOGIE pour la grandeur température

\begin{tabular}{|c|c|}
\hline Phase opérationnelle & $\begin{array}{l}\text { Equipements } \\
\text { critiques }\end{array}$ \\
\hline \multicolumn{2}{|l|}{ Phase préanalytique } \\
\hline $\begin{array}{l}\text { Transport des ovocytes du } \\
\text { bloc au laboratoire }\end{array}$ & $\begin{array}{l}\text { Malettes de } \\
\text { transport+ sondes } \\
\text { indépendantes }\end{array}$ \\
\hline \multicolumn{2}{|l|}{ Phase analytique } \\
\hline Phase ovocytaire & $\begin{array}{l}\text { Table de travail } \\
\text { chauffante }\end{array}$ \\
\hline Phase embryonnaire & $\begin{array}{l}\text { Microscope à platine } \\
\text { chauffante }\end{array}$ \\
\hline Conservation des réactifs & Réfrigérateur \\
\hline $\begin{array}{l}\text { Culture et Développement } \\
\text { des embryons }\end{array}$ & $\begin{array}{l}\text { Etuves+sondes } \\
\text { indépendantes }\end{array}$ \\
\hline \multicolumn{2}{|l|}{ Phase post analytique } \\
\hline $\begin{array}{l}\text { Conservation des } \\
\text { embryons surnuméraires }\end{array}$ & $\begin{array}{l}\text { Cuves de stockage } \\
\text { Azote liquide }\end{array}$ \\
\hline
\end{tabular}

\section{Détermination des EMT}

Après avoir recensé les équipements critiques nécessitant un suivi métrologique, nous avons défini les exigences métrologiques spécifiées, EMT c'est-à-dire les tolérances ou écarts maximum tolérés, les niveaux d'incertitude et plages d'utilisation des équipements selon les spécifications de méthodes ( données fournisseurs, textes réglementaires).

\section{Figure 3. EMT acceptables au laboratoire}

\begin{tabular}{|c|c|}
\hline Equipements & EMT acceptables \\
\hline $\begin{array}{c}\text { Etuve/Platine } \\
\text { chauffante/Table de } \\
\text { travail chauffante/ } \\
\text { malettes de transport }\end{array}$ & $37 \pm 0,5^{\circ} \mathrm{C}$ (Arrêté du \\
$03 / 08 / 10)$
\end{tabular}

\section{Définir la politique métrologique}

La politique métrologique permet l'identification du type de raccordement métrologique utilisé, la mise en place d'un planning d'étalonnage afin de permettre aux résultats d'être traçables par rapport aux unités du Système International (SI) selon les recommandations du SH REF 02

\section{Figure 4 Programme d'étalonnage}

\begin{tabular}{|l|l|l|}
\hline $\begin{array}{l}\text { Phase } \\
\text { opérationnelle }\end{array}$ & $\begin{array}{l}\text { Raccordement } \\
\text { interne ou } \\
\text { externe }\end{array}$ & $\begin{array}{l}\text { Périodicité } \\
\text { d' étalonnage } \\
\text { /vérification }\end{array}$ \\
\hline $\begin{array}{l}\text { Enceintes } \\
\text { thermiques } \\
\text { contenant les } \\
\text { embryons }\end{array}$ & interne & $\begin{array}{l}\text { Cartographie } \\
\text { initiale - ensuite } \\
1 \text { fois/an }\end{array}$ \\
\hline $\begin{array}{l}\text { Autres } \\
\text { enceintes } \\
\text { thermiques }\end{array}$ & externe & $\begin{array}{l}\text { Cartographie } \\
\text { initiale - ensuite } \\
1 \text { fois/5 ans }\end{array}$ \\
\hline $\begin{array}{l}\text { Etalon de } \\
\text { Température }\end{array}$ & externe & 1 an \\
\hline $\begin{array}{l}\text { Sonde de } \\
\text { Température }\end{array}$ & interne & 1 an \\
\hline
\end{tabular}

Le laboratoire a consigné par écrit ses raccordements métrologiques, modes opératoires, fréquence, le type de raccordements internes et externes sous la forme d'un programme d'étalonnage approprié.

- Lors d'un raccordement en externe, le laboratoire fait appel à un laboratoire d'étalonnage, accrédité pour 1' équipement et la grandeur concernée selon la norme ISO/CEI 17025[1]

-Si l'équipement ne peut être déplacé, le laboratoire vérifie que l'organisme est bien accrédité pour les étalonnages sur site ( c'est le cas des cartographies); Les cartographies permettent de tester l'homogénéité et la stabilité de l'enceinte et permet de définir les zones d'utilisation conformes aux spécifications fixées.

Aucun point ne doit être en dehors des EMT acceptables ; - Si l'équipement est déplacé, le laboratoire dans ce cas demande au prestataire qu'il s'engage et garantisse les conditions de transport adéquates, et à réception le 
laboratoire pratique un contrôle intermédiaire lorsque cela est réalisable.

-Lors d'un raccordement en interne, les aspects techniques répondent aux exigences de la norme ISO/CEI 17025 [1]; un certificat d'étalonnage (constat de vérification) est émis par le laboratoire pour son propre compte. [3]

Dans tous les cas, après un étalonnage le laboratoire vérifie l'adéquation de son équipement à ses EMT au regard de l'incertitude. [4] ; La somme de l'erreur de justesse et de l'incertitude d'étalonnage doit être inférieure au quart de l'EMT.

\section{Suivi métrologique}

Ce suivi métrologique est d'autant plus important que nous n'avons pas de CIQ ou d'EEQ pour nous alarmer sur une dérive métrologique.

Les difficultés et les risques associés à prendre en compte:

- définir des périodes de temporisation qui ne doivent pas être trop courtes pour éviter les alarmes intempestives ou trop longues pour masquer une dérive de température - choisir du matériel suffisamment performant pour répondre à l'exigence : L'erreur de justesse plus l'incertitude d'étalonnage inférieure au quart de l'EMT. -maintenir tout au long des manipulations une température de $37^{\circ} \mathrm{C}+/-0,5^{\circ} \mathrm{C}$ et prévenir les éventuelles dérives, l'étude d'impact étant difficile à évaluer

\section{Conclusion}

La fonction de métrologue est devenue une fonction clés dans le laboratoire de PMA, la métrologie s'est installée et devient indispensable pour assurer la qualité des résultats d'analyse; celle ci est mise en oeuvre de la phase pré analytique à la phase post analytique et ne couvre pas seulement l'activité analytique.

La maîtrise métrologique de nos équipements passe par 5 points fondamentaux :

- Une politique métrologique cohérente

- Une formation métrologique du personnel

- Une maîtrise documentaire

- La traçabilité de l'étalonnage et du raccordement

- La maîtrise des performances analytiques.

\begin{tabular}{|c|c|}
\hline $\begin{array}{l}\text { Les différents } \\
\text { équipements }\end{array}$ & $\begin{array}{l}\text { Suivi métrologique des } \\
\text { températures }\end{array}$ \\
\hline $\begin{array}{l}\text { Les systèmes de transport } \\
\text { (malettes) }\end{array}$ & $\begin{array}{l}\text { Détermination de la } \\
\text { température en début et } \\
\text { fin de transport avec une } \\
\text { sonde raccordée. }\end{array}$ \\
\hline $\begin{array}{l}\text { Tables chauffantes, } \\
\text { microscopes à platine } \\
\text { chauffante }\end{array}$ & $\begin{array}{l}\text { Détermination de la } \\
\text { température dans une } \\
\text { goutte de milieu de } \\
\text { culture avec une sonde } \\
\text { raccordée ; réglage de la } \\
\text { température de consigne } \\
\text { en fonction. } \\
\text { Contrôle hebdomadaire. }\end{array}$ \\
\hline Température ambiante & $\begin{array}{l}\text { Contrôle de température } \\
\text { quotidien : } \\
\text { enregistrement continu } \\
\text { des températures ou à } \\
\text { minima relevé des } \\
\text { températures minimale et } \\
\text { maximale avec un } \\
\text { thermomètre raccordé }\end{array}$ \\
\hline $\begin{array}{l}\text { Réfrigérateur; } \\
\text { congélateur, étuve }\end{array}$ & $\begin{array}{l}\text { Enregistrement continu } \\
\text { des températures avec un } \\
\text { thermomètre raccordé; } \\
\text { une } \\
\text { alarme se déclenche en } \\
\text { cas de dérive des } \\
\text { températures après } \\
\text { temporisation }\end{array}$ \\
\hline $\begin{array}{l}\text { Cartographie des enceintes } \\
\text { thermiques }\end{array}$ & $\begin{array}{l}\text { effectué avec des sondes } \\
\text { raccordées }\end{array}$ \\
\hline
\end{tabular}

\section{Références}

[1] EN ISO/IEC 17025, Exigences générales concernant la compétence des laboratoires d'étalonnage et d'essais, septembre 2005.

[2] NORME EN ISO 15189, Laboratoires d'analyses de biologie médicale. Exigences concernant la qualité et la compétence, Version 2012.

[3] Cofrac- SH GTA 04-Rev 01-Guide technique d'accréditation de vérification (portée A) / validation (portée B) des méthodes de biologie médicale-Section santé humaine- Avril 2015

[4] Cofrac- SH GTA01-Rev 01-Guide technique d'accréditation en biologie médicale- Section santé humaine. Avril 2015-

[5] Cofrac- SH REF 02- rév 03- Recueil d'exigences spécifiques pour l'accréditation des laboratoires debiologie médicale. Section santé humaine. Septembre 2013

[6] Arrêté du 11 avril 2008 relatif aux régles de bonnes pratiques cliniques et biologiques d'assistance médicale à la procréation .version consolidée du 12 juin 2014 